Proceedings of the 16th Czech and Slovak Conference on Magnetism, Košice, Slovakia, June 13-17, 2016

\title{
LSMO/YBCO Heterostructures and Investigation of "Negative" Resistance Effect in the Interface
}

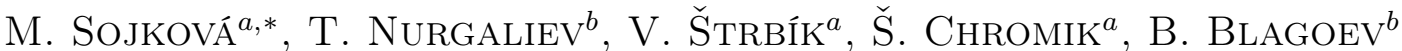 \\ AND M. ŠPANKOVÁa \\ ${ }^{a}$ Institute of Electrical Engineering, Slovak Academy of Sciences, Dúbravská cesta 9, 84104 Bratislava, Slovakia \\ ${ }^{b}$ Institute of Electronics, Bulgarian Academy of Sciences, 72 Tsarigradsko Chausse, 1784 Sofia, Bulgaria
}

Samples containing the ferromagnetic manganite $\mathrm{La}_{0.67} \mathrm{Sr}_{0.33} \mathrm{MnO}_{3}$ (LSMO) and high temperature superconducting $\mathrm{YBa}_{2} \mathrm{Cu}_{3} \mathrm{O}_{7}$ (YBCO) single thin film areas and $\mathrm{YBCO} / \mathrm{LSMO}$ bilayer area were prepared on $\mathrm{LaAlO} 3$ (LAO) substrates and were used for investigation of the electrical properties of the interface. The measurements in the YBCO/LSMO interface demonstrated "negative" values of the resistance. A good interpretation of the obtained results was performed in the framework of a $1 \mathrm{D}$ model, which took into account the resistance of the interface $R_{i f}$ and the temperature dependence of the resistance of YBCO and LSMO films. It was shown that the effect of "negative" resistance arises because of the redistribution of the measuring electrical current in the interphase area if the resistance of the interface $R_{i f}$ is small in comparison with the resistances of the neighboring electrodes.

DOI: 10.12693/APhysPolA.131.842

PACS/topics: 73.40.-c, 73.40.Cg, 74.78.Fk, 74.72.--h, 75.50.Cc

\section{Introduction}

Combinations of ferromagnetic (FM) perovskite manganite and high temperature superconducting (HTS) thin films are of great interest currently both in connection with their attractive physics, originated from the competition of superconductivity and ferromagnetism in the interface, and with their potential application in various spintronics devices [1]. In spite of the conducted intensive researches during the last decade, the electrical properties of such thin film structures remain still far from full understanding. Fabrication of HTS/manganite heterostructures and investigation of their electric characteristics in different areas of the sample and in the interface are the aims of this paper.

\section{Experimental}

Heterostructures consisting of high quality perovskite ferromagnetic manganite $\mathrm{La}_{0.67} \mathrm{Sr}_{0.33} \mathrm{MnO}_{3}$ and HTS $\mathrm{YBa}_{2} \mathrm{Cu}_{3} \mathrm{O}_{7}$ thin films of thickness about $50 \mathrm{~nm}$ were deposited on $\mathrm{LaAlO}_{3}$ single crystal substrates by pulsed laser deposition (PLD, an $\mathrm{KrF}$ excimer laser operating at $248 \mathrm{~nm}$ ) method. A standard photolithography process and wet etchings were used to prepare the samples of necessary configurations, suitable for performance of DC resistive measurements.

The samples contained HTS YBCO meander and FM LSMO strip with widths of $0.5 \mathrm{~mm}$ crossing each other at $\approx 90^{\circ}$ (see for example [2]). The temperature dependences of the resistance ( $R$ vs. $T$ dependence) of the strips and of the interface in the crossing area were measured using a DC four probe method and analyzed in terms of the interaction of the HTS and FM layers.

*corresponding author; e-mail: michaela.sojkova@savba.sk

\section{Results}

Figure 1 shows temperature dependences of resistance of HTS YBCO and FM LSMO strips. A small "step" in $R$ vs. $T$ dependence of LSMO film, observed at $T \approx 85 \mathrm{~K}$, is stimulated by superconducting transition of YBCO film in the crossover area. One can see some difference between the critical temperatures $T_{c}$, estimated from the $R-T$ dependences of YBCO and LSMO strips, and it is by several $\mathrm{K}$ higher in the later case. This means that $T_{c}$ of YBCO film is higher in the crossover (or bilayer) area of the sample, where HTS film is deposited on the bottom of LSMO layer.

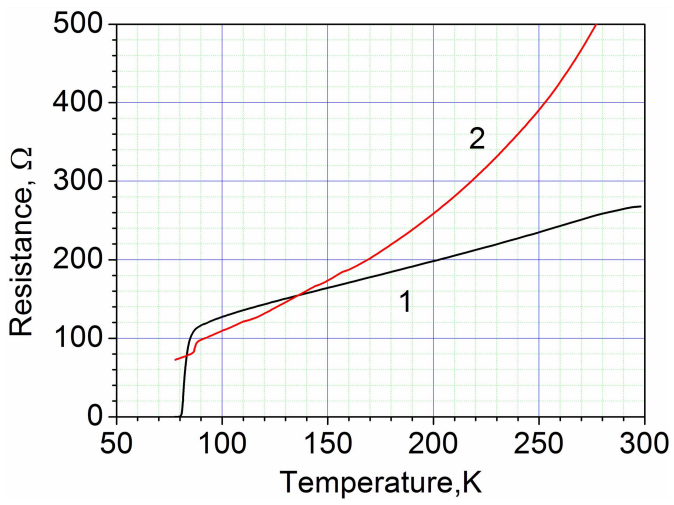

Fig. 1. Temperature dependence of the resistance of YBCO (curve 1) and LSMO (curve 2) strips crossing each other at $\approx 90^{\circ}$.

Temperature dependence of the resistance of the interface between YBCO and LSMO strips, measured by four probe method is shown in Fig. 2. It is interesting to note that the resistance is "negative" in the whole temperature range of measurements. Critical temperature, determined from the temperature dependence of the "negative" resistance is in a good agreement with $T_{c}$ found from the 


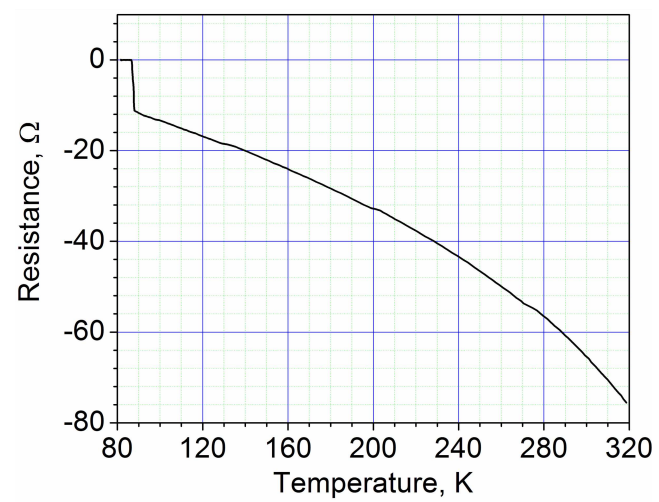

Fig. 2. Temperature dependence of the interface resistance between the YBCO meander and LSMO strips measured by four probe method. The resistance is "negative" in the whole temperature range.

step-like dependence of $R$ vs. $T$, which is observed in LSMO strip. This confirms again that the bilayer area of the sample is characterized by higher value of $T_{c}$ since the LSMO film serves for YBCO as a seed layer with better lattice matching than the LAO substrate.

\section{Theoretical consideration and discussion}

For better understanding of the observed "negative" resistance in our measurements, some theoretical aspects of the effect were analyzed. Profile of the structure, consisting of thin films 1, 2, separated by a thin interface layer is schematically shown in Fig. 3. Our investigation is based on 1D approximation, considered in [3] for a tunneling sample.

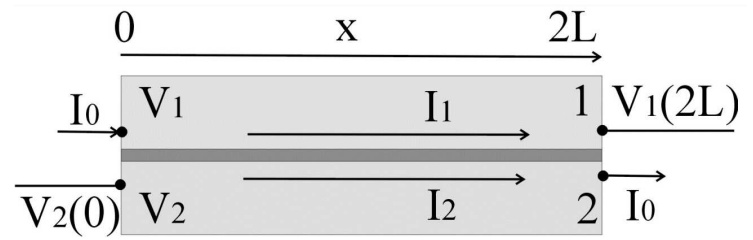

Fig. 3. Profile of the structure, consisting of thin films 1,2 , separated by a thin interface layer.

The currents $I_{1}(x), I_{2}(x)$ and voltages $V_{1}(x)$ and $V_{2}(x)$ along the films will be functions of the position $x$. The total resistance along the films is $R_{S}$ (film 1), $R_{F}$ (film 2 ), while the resistance across the interface is $R_{i f}$. The electrical current and voltage in the structure are approximately described by the equations

$$
\begin{aligned}
& \mathrm{d} V_{1}(x) / \mathrm{d} x=-\left(R_{S} / 2 L\right) I_{1}(x), \\
& \mathrm{d} V_{2}(x) / \mathrm{d} x=-\left(R_{F} / 2 L\right) I_{2}(x), \\
& V_{1}(x)-V_{2}(x)=2 L R_{i f}\left(\mathrm{~d} I_{2}(x) / \mathrm{d} x\right)
\end{aligned}
$$

and satisfy the boundary conditions $I_{1}(x)+I_{2}(x)=I_{0}$, $I_{1}(0)=I_{2}(2 L)=I_{0}, I_{2}(0)=0$. Here, $2 L$ is the "length" of the 1D interface. The solutions of Eqs. (1)-(3) give the voltages $V_{1}(x), V_{2}(x)$ and currents $I_{1}(x), I_{2}(x)$ for the different cross-sections of the films 1,2 in the structure. The voltages $V_{1}(x), V_{2}(x)$ can be experimentally measured and used for evaluation of the resistance of the sample and the interface. Let us consider a situation when the current-contacts are placed as in Fig. 3, and the voltage contacts, placed on the surfaces of the films 1 and 2, have the same $x$-coordinates. The resistance determined in this case $R_{e f}(x)=\left|V_{1}(x)-V_{2}(x)\right| / I_{0}$ can be considered as some effective resistance of the sample for the position $x$ of the voltage contacts. The simplest relation between the effective resistance $R_{e f}$ and the real value of the interface resistance $R_{i f}$ can be determined if film 1 is in the superconducting state $\left(R_{S}=0\right)$ :

$$
\begin{aligned}
& R_{e f}(x)=\sqrt{R_{F} R_{i f}}\left(\cosh \left(\sqrt{R_{F} / R_{i f}} y\right)\right) \\
& \quad /\left(\sinh \left(\sqrt{R_{F} / R_{i f}}\right)\right),
\end{aligned}
$$

where $y=x /(2 L)$. Figure 4 demonstrates $R_{e f}(x)$ in the logarithmic scale modeled for different positions $x$ of the measuring contacts along the structure. It can be noted existence of a simple relation between the slopes of the curves $S=\mathrm{d}\left(\ln \left(R_{e f}\right) / \mathrm{d} y\right.$ in Fig. 3 and the parameters of the structure $\sqrt{R_{F} / R_{i f}} \approx S$. Such kind of measurements could be used for evaluation of the interface resistance $R_{i f}$ if the structure is "long" enough and $R_{F}$ is known: $R_{i f} \approx R_{F} / S^{2}$.

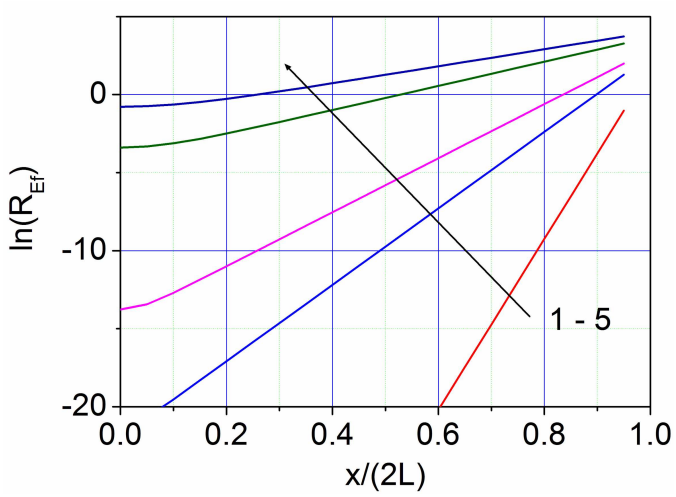

Fig. 4. Effective resistances of the sample in the logarithmic scale modeled for different positions $x$ of the measuring contacts along the structure. $R_{F}=300 \Omega$, $R_{i f}=0.1,0.5,1,5,10 \Omega$ (curves $1-5$, respectively).

One can also try to evaluate the interface resistance from the four-probe measurement of the resistance $R_{0-2 L}$ (the positions of the current- and voltage-contacts are shown schematically in Fig. 3). The solution of above equations for such a situation can be obtained in the following form:

$$
\begin{aligned}
& R_{0-2 L}=\left(V_{1}(2 L)-V_{2}(0)\right) / I_{0}=\frac{R_{i f}^{1 / 2}}{\left(R_{F}+R_{S}\right)^{3 / 2}} \\
& \quad \times \frac{1}{\sinh (2 k L)}\left(R_{F}^{2}+R_{S}^{2}+2 R_{S} R_{F} \cosh (2 k L)\right) \\
& \quad-\frac{R_{F} R_{S}}{R_{F}+R_{S}},
\end{aligned}
$$

where $k=\sqrt{\left(R_{F}+R_{S}\right) / R_{i f}} /(2 L)$.

Analysis shows that if $R_{F}, R_{S} \gg R_{i f}$, the formula (5) can give "negative" values of the resistance arising from 
the effect of redistribution of the measuring electrical current in the interphase area.

An experimental $R_{0-2 L}(T)$ dependence (measured in YBCO/LSMO bilayer) and the examples of modeling of $R_{0-2 L}(T)$ using formula (5) are shown in Fig. 5. The critical temperature $T_{c}$ and the resistances of single superconducting and manganite films determined from the experiments (see Fig. 1) were used with the fitting coefficients $1.06,0.17$ and 0.71 for modeling (i.e. $T_{c}=1.06 T_{C \exp }, R_{F}=0.17 R_{F \exp }$ and $\left.R_{S}=0.71 R_{S \exp }\right)$ of $R(T)$ dependence in the bilayer part of the structure.

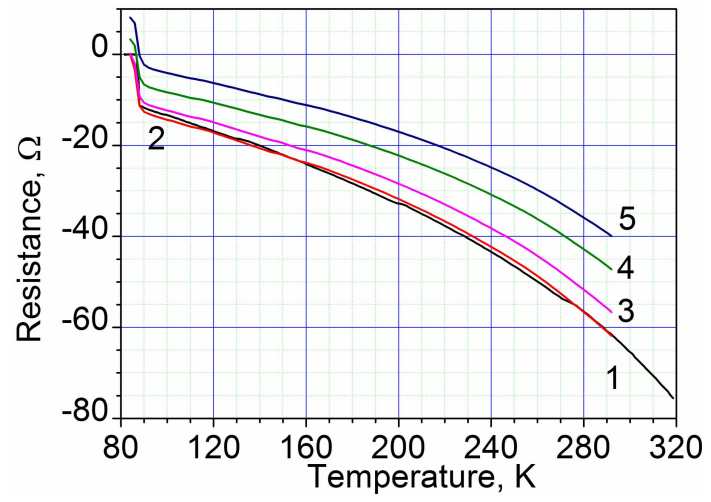

Fig. 5. The temperature dependences of the resistance between the contacts attached at $x=2 L$ (film 1 ) and $x=0$ (film 2). Curve 1 demonstrates the dependence $R_{0-2 L}(T)$ measured in a HTS/FM bilayer. Curves 2-5 are the $R_{0-2 L}(T)$ dependences modeled for the values of the interface resistance $R_{i f}=0.1,1,5,10 \Omega$, respectively. The other parameters of modeling are presented in the text.

It can be seen that the modeled resistance is "negative", its behavior is the same as that of the experimental $R_{0-2 L}$ vs. $T$ dependence at $T<T_{c}$. This resistance is not strongly sensitive to $R_{i f}$ at $R_{F}, R_{S} \gg R_{i f}$. When one of the films is in superconducting state (i.e. $T<T_{c}$ and $R_{S}=0$ ), the relation between the measured resistances $R_{0-2 L}$ and the resistances of the second film and the interface $\left(R_{F}\right.$ and $\left.R_{i f}\right)$ is given by the formula

$$
R_{0-2 L}=\sqrt{R_{F} R_{i f}} / \sinh \left(R_{F} / R_{i f}\right)^{1 / 2} .
$$

In this case the resistance $R_{0-2 L}$ is positive and significantly smaller than the interface resistance $R_{i f}$. For example, an estimation using the above formula gives $R_{0-2 L} / R_{i f} \approx 10^{-6}$ for standard LSMO films $\left(R_{F}=\right.$ $300 \Omega$ ) if even the interface resistance is assumed to be not too small $R_{i f}=1 \Omega$. For higher values of $R_{i f}$, the whole $R_{0-2 L}(T)$ dependence is "shifted" gradually to the positive values with increase of $R_{i f}$ (Fig. 5, curves 2-5). It can be seen from formulae (4) and (6) that both above measurement schemes could give the real value of the interface resistance (i.e. $R_{e f}=R_{i f}$ and $R_{0-2 L}=R_{i f}$ ) if we assume hypothetically that the components 1 and 2 of the bilayer are in superconducting state $\left(R_{S}=0\right.$, $\left.R_{F}=0\right)$. Actually, in the case of sample with increased $R_{i f}$ value the experimental observed $R_{0-2 L}(T)$ dependence was shifted to positive values (Fig. 6).



Fig. 6. The temperature dependence of $R_{0-2 L}$ observed in an YBCO/LSMO sample with increased value of the interface resistance $R_{i f}$. The shift of the dependence to positive values is evident.

\section{Conclusions}

Samples containing the FM manganite LSMO and HTS YBCO single thin film areas (with the thickness of $\approx 50 \mathrm{~nm}$ ) and $\mathrm{YBCO} / \mathrm{LSMO}$ bilayer area was prepared on $\mathrm{LaAlO}_{3}$ (LAO) substrates and were used for investigation of the electrical properties of the interface. Four probe measurements demonstrated "negative" values of the interface resistance. A good interpretation of the obtained results was performed in the framework of $1 \mathrm{D}$ model, which took into account the resistance of the interface $R_{i f}$ and the temperature dependence of the resistance of YBCO and LSMO films $R_{S}$ and $R_{F}$. It was shown that the effect of "negative" resistance arises because of the redistribution of the measuring electrical current in the interphase area if the resistance of the interface $R_{i f}$ is small in comparison with the resistances of the neighboring electrodes. For higher values of $R_{i f}$, the measured value of the resistance $R_{0-2 L}(T)$ can "shift" to the positive values with increase of $R_{i f}$.

\section{Acknowledgments}

This work was supported by the Joint Research Project "Perovskite heterostructures of nanometric thickness for sensors and spintronics" between the Institute of Electronics BAS, Bulgaria and the Institute of Electrical Engineering SAS, Slovakia, by the project VEGA No. 2/0120/14 and projects APVV-0494-11, APVV-140613, APVV LPP-0078-07 and "CENTE II" ITMS code 26240120019.

\section{References}

[1] M.E. Flatté, IEEE Trans. Electron. Dev. 54, 907 (2007).

[2] M. Sojková, V. Štrbík, T. Nurgaliev, Š. Chromik, E. Dobročka, M. Śpanková, B. Blagoev, N. Gál, J. Phys. Conf. Series 700, 012022 (2016).

[3] I. Giaver, in Tunneling Phenomena in Solids, Eds. E. Burstein, S. Lundqvist, Plenum Press, New York 1969, p. 19 